\title{
Academic partnerships 2014
}

\section{Brady Huggett}

The number of disclosed partnerships for academic institutions declined significantly in 2014, down to 278 from 358 in 2013 (457 in 2012). Oncology deals again led the way (56), with neurology (35) and infectious disease (31) following. The large university systems (California and Texas,

respectively) were the most active schools (Table 1); the University of Oxford is tied with The Broad Institute with five deals each. AstraZeneca was again the most active pharma, forming 18 partnerships with academic and research institutions (Fig. 1), up from 14 in 2013.

\section{Table 1 Academic partnerships 2014}

\begin{tabular}{ll} 
University & Partners \\
\hline $\begin{array}{l}\text { University of California } \\
\text { system (11 deals) }\end{array}$ & $\begin{array}{l}\text { UC Irvine (UCI), Broad Institute of MIT and Harvard, Harvard Medical School, } \\
\text { Icahn School of Medicine at Mount Sinai, National Institutes of Health (NIH), } \\
\text { Oregon Health and Science University (OHSU) }\end{array}$ \\
\hline & UCI, California Stem Cell, California Institute for Regenerative Medicine (CIRM) \\
\hline & $\begin{array}{l}\text { University of California San Francisco (UCSF), Cornell University, Lawrence } \\
\text { Livermore National Laboratory, New York University, US Department of Defense (DoD) }\end{array}$ \\
\hline $\begin{array}{l}\text { University of California San Diego (UCSD), University of California Santa Cruz } \\
\text { (UCSC), Illumina, Salk Institute, Scripps Research Institute, Stanford University, }\end{array}$ \\
CIRM, J. Craig Venter Institute \\
\hline UCSF, Quest Diagnostics \\
\hline UCSF, Advaxis \\
\hline UCSF, Daiichi Sankyo \\
UCSD, PaxVax \\
UCSF, AstraZeneca (MedImmune) \\
University of California Los Angeles, OncoSec Medical, PerkinElmer \\
UCSF, OncoSec Medical
\end{tabular}

Terms

$\$ 64$ million NIH grant to create the Library of Integrated Network-based Cellular Signatures, a database of human cellular responses. The data will be freely available.

\$4.5 million CIRM grant to develop human stem cell-derived transplantable 3D retinal tissue to treat incurable retinal diseases.

$\$ 26$ million, five-year grant from US DoD Defense Advanced Research Projects Agency (DARPA) to understand and heal disrupted brain circuitry to treat mental illnesses.

$\$ 40$ million CIRM grant for a Center of Excellence in Stem Cell Genomics with Stanford and the Salk Institute as co-principal investigators, UCSD, Scripps, the $J$. Craig Venter Institute and Illumina as collaborators; UCSC handles data. Develop precision medicine diagnostics in autism, oncology, neurology; women's health. Combine Advaxis' immunotherapy platform with UCSF targets in prostate cancer. Focus on drugs and diagnostics for neurodegenerative diseases. Combination vaccine to prevent genital herpes simplex virus infection. Three-year partnership to discover and develop small molecules and biologics. Partnership on molecular biomarkers for cancer immune responses. Evaluate Keytruda (pembrolizumab) with Merck's anti-PD-1 therapy and OncoSec's ImmunoPulse (intratumoral IL-12) in metastatic melanoma.

University of Texas MD Anderson Cancer Center, Johnson \& Johnson (J\&) (Boston Innovation Center (8 deals) and Janssen Biotech)

MD Anderson Cancer Center, J\&J (Boston Innovation Center and Janssen Biotech) MD Anderson Cancer Center, Pfizer (Rinat Biotech)

MD Anderson Cancer Center, Manhattan Scientifics

Three-year deal to develop personalized immunotherapies for cancer through MD Anderson's Moon Shots Program.

Partnership to develop personalized immunotherapies for cancer.

Three-year deal on cancer immunotherapies (Moon Shots).

Validate a non-invasive technology, nanoMRX, in detecting cancer.

Three-year deal on cancer immunotherapies (Moon Shots). Develop immunotherapies for cancer (Moon Shots).

MD Anderson Cancer Center, GlaxoSmithKline

MD Anderson Cancer Center, Bristol-Myers Squibb

UT Medical Branch in collaboration with Scripps Research Institute and 10 others

Broad Institute of MIT Merck (EMD Serono), Pfizer

and Harvard (5 deals)

Tetra Discovery Partners

Emerald Bio, NanoRacks, Protein BioSolutions, Center for the Advancement of Science in Space (CASIS)

Harvard Medical School, Icahn School of Medicine at Mount Sinai, NIH, OHSU, UCl

Amgen, Massachusetts General Hospital

University of Oxford GlaxoSmithKline, Medical Research Council, NIH, Wellcome Trust

(5 deals)

BioMotiv, The Harrington Discovery Institute (University Hospitals, Cleveland)

Bayer HealthCare

Abide Therapeutics

VolitionRx
Partnership to evaluate Opdivo (nivolumab), Yervoy (ipilimumab) and three undisclosed clinical-stage immuno-oncology compounds.

Five-year NIAID \$28 million award to a consortium led by Scripps to develop immunotherapies for filoviruses and arenaviruses.

Partnership to identify biomarkers to define target patient populations for systemic lupus erythematosus (SLE) and lupus nephritis.

Partnership to evaluate the ability of the company's phosphodiesterase-4D (PDE-4D) inhibitor to improve cognition in schizophrenia.

Explore microgravity on crystallization of proprotein convertase subtilisin/kexin type 9 (PCSK9) and myeloid leukemia cell differentiation protein (MCL1).

$\$ 64$ million NIH grant to create the Library of Integrated Network-based Cellular Signatures (LINCS), a database of human cellular responses. Explore targets, therapies for inflammatory bowel disease.

$£ 2.8$ million ( $\$ 4.7$ million) grant from Wellcome Trust, Medical Research Council and the UK's Department for International Development for an Ebola vaccine. International initiative to drive early-stage drug discovery and development. Partnership for therapies to treat endometriosis and uterine fibroids. Collaboration on the therapeutic potential of serine hydrolases.

Assess VolitionRx's proprietary Nucleosomics platform to diagnose endometriosis.

University of Edinburgh, Stanford, Yale and Columbia Universities, three deals each; Medical University of South Carolina, two deals. Because of consortia, some deals appear more than once. Source: BioCentury.

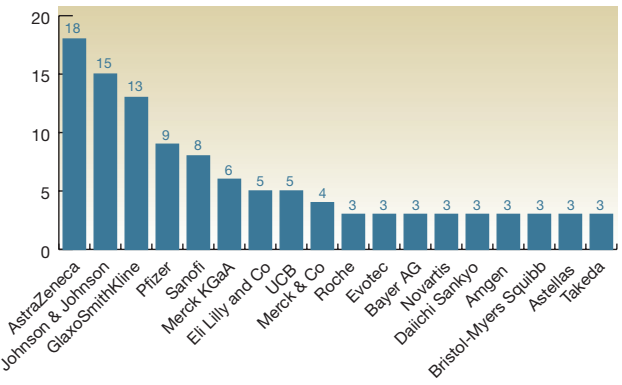

Figure 1 Most active pharma, biotech with academic/research institutes, 2014. Source: BioCentury.

\section{First Rounders Podcast:} Kari Stefansson

Kari Stefansson is the founder and CEO of deCODE genetics, based in Reykjavik, Iceland. His conversation with Nature Biotechnology covers the founding of deCODE, his love for literature (and his favorite poet), plus his encounter with the tortured chess grandmaster Bobby Fischer.

http://www. nature.com/nbt/podcast/index.html.

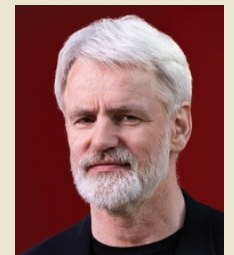

Brady Huggett is business editor at Nature Biotechnology. 